\title{
Risposte esatte ai test di verifica
}

La neurostimolazione midollare nel trattamento dell'ischemia critica non rivascolarizzabile del paziente uremico

U. Brummer, P. Cappelli, A. Piano, et al

\section{TEST DI VERIFICA - 1 - Pag 23}

Domanda n. 1: risposta esatta B
b) $25 \%$

Domanda n. 2: risposta esatta D

d) danno delle corna o delle radici spinali

Domanda n. 3: risposta esatta D

d) tutte le precedenti

Insufficienza renale cronica in un paziente coinfetto HIV/HCV tossicodipendente. Un iter diagnostico-terapeutico complesso. “... Lasciate ogne speranza, voi ch'intrate” ?

C. Cherubini, S. Papalini

\section{TEST DI VERIFICA - 2 - Pag 28}

Domanda n. 1: risposta esatta B

b) glomerulosclerosi focale, nefrite interstiziale

Domanda n. 2: risposta esatta C

c) le metodiche sostitutive si equivalgono

Domanda n. 3: risposta esatta B

b) gli inibitori nucleosidici della trascrittasi inversa

Domanda n. 4: risposta esatta $A$

a) analoga

L'utilizzo degli inibitori del segnale di proliferazione (PSI) nel trapianto renale come terapia di conversione. Indicazioni e possibili complicanze

A. Rosati, E. Bertoni, A. Larti, et al

\section{TEST DI VERIFICA - 3 - Pag 38}

Domanda n. 1: risposta esatta C

c) La sospensione di Ciclosporina o Tacrolimus e l'introduzione di Rapamicina o Everolimus ottengono spesso un miglioramento o stabilizzazione della funzione renale.

Domanda n. 2: risposta esatta A

a) Riduzione/ sospensione degli inibitori delle calcineurine e degli agenti antiproliferativi, introduzione di Sirolimus o Everolimus.

d) E mivita: Everolimus ha una emivita più breve del Sirolimus ( 28 vs 60 ore). 